\title{
Un ciclo de mejora en consultas de optometría
}

\section{A cycle of improvement in optometry consultations}

María del Carmen Sánchez González.

Departamento de Física de la Materia Condensada.

Facultad de Farmacia. Universidad de Sevilla. msanchez77@us.es

Orcid: https://orcid.org/0000-0002-9453-8615

DOI: http://dx.doi.org/10.12795/9788447231003.063

Pp.: 1333-1347 


\section{Objetivo}

El Ciclo de Mejora en el aula (CIMA) tiene como objetivo que los alumnos sepan llegar a un diagnóstico de una disfunción visual, a partir de los valores de cada una de las variables que definen tanto el estado de la función acomodativa como vergencial. Han de interpretar las variables, relacionarlas, emitir un diagnóstico, una solución y prevención del caso clínico que se plantea.

\section{Contexto}

El Ciclo de Mejora en el aula (CIMA) se ha planteado durante el curso 2020-21 en un grupo de prácticas de la asignatura "Consulta de Optometría. Casos clínicos especiales" del Grado de Óptica y Optometría de la Universidad de Sevilla.

Las prácticas se dividen en 10 sesiones con 8 alumnos por grupo. Cada sesión tiene una duración de 4 horas. El CIMA se implementó durante dos prácticas sucesivas dividiendo el grupo en dos subgrupos de 4 alumnos cada uno. El reducido número de alumnos facilitó el trabajo en equipo y la interacción entre ellos. El desarrollo del CIMA siguió una metodología de investigación basada en problemas y casos prácticos.

Al ser alumnos del último curso tenían conocimientos previos y motivación que les hizo implicarse en la dinámica de la asignatura. Se consiguió un excelente ambiente de trabajo y alto grado de participación.

Ciclos de Mejora en el Aula (2020). Experiencias de Innovación Docente de la US Esta obra se distribuye con la licencia Creative Commons 


\section{Diseño del CIMA}

\section{Principios didácticos}

Las estrategias formativas que desarrollé durante la puesta en práctica del CIMA podemos calificarla de la siguiente forma:

- Aprendizaje autónomo del estudiante. Como parte de la orientación basada en proyectos, los estudiantes debían buscar, ordenar y clasificar información, comprenderla, representarla y exponerla de forma que demuestren una adecuada comprensión. El estudiante debe asimismo leer y comprender textos necesarios para el desarrollo de la tarea. Este aprendizaje autónomo se lleva a cabo en los momentos de trabajo individual y en grupo para dar respuesta a la situación planteada cada semana.

- Enseñanza directa. Por mi parte, como docente presento a los estudiantes los conceptos, teorías y principios que considero que el estudiante debe adquirir. Para ello recurro al uso de presentaciones y videos.

- Aprendizaje basado en proyectos. La enseñanza se organiza en torno a un proyecto que los estudiantes deben de desarrollar a lo largo de las dos semanas que dura el CIMA (Porlan et al., 2017).

- Aprendizaje colaborativo. Como consecuencia de la estrategia anteriormente descrita, los estudiantes deben de aprender a colaborar para la resolución de las situaciones prácticas que en cada momento se les presentan. Han de mostrar avances de sus trabajos durante las dos semanas que dura el CIMA.

Ciclos de Mejora en el Aula (2020). Experiencias de Innovación Docente de la US Esta obra se distribuye con la licencia Creative Commons 


\section{Cuestionario}

En el cuestionario (Tabla 1), planteo el concepto de disfunciones acomodativas y binoculares no estrábicas. Para ello introduzco un problema muy común en consulta consecuencia de la actual situación, " Síndrome visual informático (SVI)".

El SVI es una alteración temporal consecuencia del uso ininterrumpido de dispositivos digitales. Esta situación implica un sobreesfuerzo continuo de la acomodación y vergencia y altera la eficiencia del sistema visual, apareciendo síntomas astenópicos que incluyen visión borrosa, dificultad en la lectura, diplopía y frecuentemente imposibilidad de mantener una visión confortable durante un tiempo prolongado.

A través del concepto SVI, explicaré los mecanismos de acomodación y vergencia, variables que definen acomodación y visión binocular, técnicas de medida, diagnóstico de disfunciones acomodativas y binoculares no estrábicas, prevención y estrategias terapéuticas.

En el cuestionario planteo preguntas acerca del "saber", "saber hacer" y "actitud".

Tabla 1. Cuestionario

\begin{tabular}{|l|l|l|}
\hline Preguntas & Evaluación inicial & Evaluación final \\
\hline SABER & & \\
\hline ¿Qué es la acomodación? & & \\
\hline ¿Qué es la visión binocular? & & \\
\hline ¿Para qué sirve la acomodación & & \\
\hline $\begin{array}{l}\text { ¿Qué es necesario para que ocurra } \\
\text { visión binocular? }\end{array}$ & & \\
\hline $\begin{array}{l}\text { ¿Qué variables definen la } \\
\text { acomodación? }\end{array}$ & & \\
\hline $\begin{array}{l}\text { ¿Qué variables definen la visión } \\
\text { binocular? }\end{array}$ & & \\
\hline
\end{tabular}

Ciclos de Mejora en el Aula (2020). Experiencias de Innovación Docente de la US Esta obra se distribuye con la licencia Creative Commons 


\begin{tabular}{|l|l|l|}
\hline $\begin{array}{l}\text { ¿Qué tipo de disfunciones se } \\
\text { asocian a la acomodación y } \\
\text { vergencias? }\end{array}$ & & \\
\hline SABER HACER & & \\
\hline $\begin{array}{l}\text { ¿ Qué procedimientos llevarías a } \\
\text { cabo para valorar la acomodación? }\end{array}$ & & \\
\hline $\begin{array}{l}\text { ¿ Qué procedimientos llevarías a } \\
\text { cabo para valorar la visión binocular? }\end{array}$ & & \\
\hline $\begin{array}{l}\text { ¿ Cómo se interpretan los valores } \\
\text { normativos? }\end{array}$ & & \\
\hline $\begin{array}{l}\text { ¿ Cómo se diagnostican las } \\
\text { disfunciones relacionadas con la } \\
\text { visión binocular y acomodación? }\end{array}$ & & \\
\hline $\begin{array}{l}\text { ¿ Cómo se clasifican las disfunciones } \\
\text { acomodativas y binoculares? }\end{array}$ & & \\
\hline ACTITUD & & \\
\hline $\begin{array}{l}\text { ¿ Cómo se debe proceder con el } \\
\text { paciente durante la exploración? }\end{array}$ & & \\
\hline $\begin{array}{l}\text { ¿ Cómo se previenen las } \\
\text { disfunciones relacionadas con el } \\
\text { sistema visual? }\end{array}$ & & \\
\hline
\end{tabular}

\section{Mapa de contenidos}

En el mapa de contenidos (figura 1), distingo "los contenidos nucleares" (concepto de disfunciones acomodativas y binoculares y diagnóstico de ambas), conceptos que considero, todo óptico-optometrista ha de saber.

Además, se incluyen datos de prevalencia que nos dan idea de la relevancia social de estas disfunciones y valores normativos cuya interpretación nos conduce al diagnóstico. Ambos, datos de prevalencia y normativos los considero como "los contenidos de datos".

Por último, destaco "contenidos de valor" en forma de estrella. Entre ellos incluyo la prevención y evaluación que todo alumno de cuarto curso de óptica y optometría ya debe estar cualificado para llevar a cabo.

Ciclos de Mejora en el Aula (2020). Experiencias de Innovación Docente de la US Esta obra se distribuye con la licencia Creative Commons 


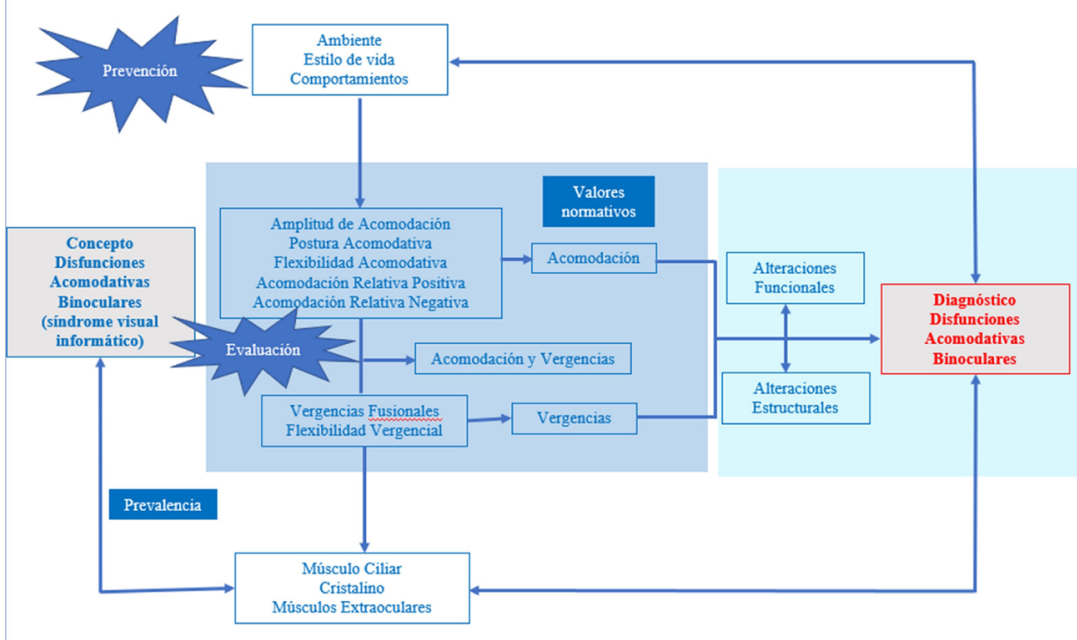

Figura 1. Mapa de Contenidos del CIMA.

\section{Aplicación del CIMA}

\section{Secuencia de actividades}

El primer día les explico que estoy realizando un curso de formación de profesorado de la Universidad de Sevilla y que parte de los "deberes" que he de realizar es implantar un Ciclo de Mejora en el aula. Les aclaro que el test inicial y final que han de cumplimentar en la primera y segunda sesión no influye en la nota, ya que es una cuestión que les preocupa. Les informo que no es necesario poner nombres y apellidos, basta con un número identificativo, hecho que les tranquiliza.

Les explico que no voy a dar una clase tradicional. Me baso en los siete principios que enumera Ken Bain (Bain, 2007) y puesto que estamos en prácticas es un entorno

Ciclos de Mejora en el Aula (2020). Experiencias de Innovación Docente de la US Esta obra se distribuye con la licencia Creative Commons 
que se adecúa a este tipo de enseñanza. No entienden muy bien que no sea yo la que comience la clase como hago siempre y que les plantee como inicio de la clase distintas cuestiones.

Diseño

Primera Sesión

Cumplimentación del Test inicial (15 min).

Presentación de la primera pregunta y dinámica a seguir (15 min). "Cómo influye la acomodación en el sistema visual" (Grupo A ) y "Cómo influye la musculatura extraocular en el sistema visual" (Grupo B). Como ayuda les defino el síndrome visual informático.

Primera ronda de actividades prácticas en torno a la primera pregunta realizada (30 min).

Puesta en común de las ideas de cada grupo y debate entre ellos (30 min).

Exposición por mi parte sobre la primera pregunta planteada, partiendo de las aportaciones de los estudiantes y estableciendo un diálogo con ellos (30 min).

Descanso (15 min).

Presentación de la segunda pregunta y dinámica a seguir (15 min). "Qué variables definen la acomodación" (Grupo A ) y "Qué variables definen la visión binocular" (Grupo B).

Segunda ronda de actividades prácticas en torno a la segunda pregunta realizada (30 min).

Puesta en común de las ideas de cada grupo y debate entre ellos (30 min).

Exposición por mi parte sobre la segunda pregunta planteada, partiendo de las aportaciones de los 
estudiantes y estableciendo un diálogo con ellos (30 $\min )$.

Segunda sesión

Presentación de la tercera pregunta y dinámica a seguir (15 min). "Cómo interpretas los valores de cada uno de los valores de las variables acomodativas que te presento" (Grupo A ) y "Cómo interpretas cada uno de los valores de las variables vergenciales que te presento" (Grupo B). Como ayuda les defino el síndrome visual informático.

Primera ronda de actividades prácticas en torno a la tercera pregunta realizada (30 $\mathrm{min}$ ).

Puesta en común de las ideas de cada grupo y debate entre ellos (30 min).

Exposición por mi parte sobre la tercera pregunta planteada, partiendo de las aportaciones de los estudiantes y estableciendo un diálogo con ellos (30 min).

Descanso (15 min).

Presentación de la cuarta pregunta y dinámica a seguir (15 min). "Emite un diagnóstico, tratamiento y prevención según valores de variables acomodativas" (Grupo A ) y "Emite un diagnóstico, tratamiento y prevención según valores de variables vergenciales" (Grupo B).

Segunda ronda de actividades prácticas en torno a la cuarta pregunta realizada (30 $\mathrm{min}$ ).

Puesta en común de las ideas de cada grupo y debate entre ellos (30 min).

Exposición por mi parte sobre la cuarta pregunta planteada, partiendo de las aportaciones de los estudiantes y estableciendo un diálogo con ellos (30 min).

Cumplimentación del test final (15 min).

Ciclos de Mejora en el Aula (2020). Experiencias de Innovación Docente de la US 


\section{Experimentación}

En cada sesión de prácticas acuden 8 alumnos a los que divido en dos grupos de 4 alumnos cada uno. Cada sesión dura 4 horas. El CIMA lo implanto a lo largo de 2 sesiones de prácticas.

La primera sesión del ciclo se centró en la influencia de la acomodación y musculatura extraocular en el sistema visual. Introduzco el término de "sindrome visual informático", tema que resulta interesante a los alumnos, de esta forma fue fácil captar su atención.

Empiezan a repasar individualmente la lectura de dos artículos que ya han trabajado en casa: "Characterization of visual symptomatology associated with refractive, accommodative, and binocular anomalies" (Cacho-Martínez, Cantó-Cerdán, Carbonell-Bonete, \& García-Muñoz, 2015)accommodative, and nonstrabismic binocular dysfunctions and to assess the association between dysfunctions and symptoms. Methods. 175 randomised university students were examined. Subjects were given a subjective visual examination with accommodative and binocular tests, evaluating their symptomatology. Accommodative and binocular dysfunctions (AD, BD y "Accommodative and binocular dysfunctions: prevalence in a randomised sample of university students" (García-Muñoz, Carbonell-Bonete, Cantó-Cerdán, \& Cacho-Martínez, 2016). Ambos artículos definen los trastornos visuales consecuencia del uso continuo de pantallas digitales. Concluyen que las anomalías acomodativas y las disfunciones binoculares no estrábicas son trastornos de la visión consecuencia de esta situación, que afectan a la binocularidad y rendimiento visual de los sujetos. Además, estas disfunciones tienden a provocar dificultades en la realización de actividades relacionadas con la visión próxima con síntomas que pueden incluir visión borrosa, dificultad en la lectura, dolor de cabeza, diplopía y en muchos casos imposibilidad de mantener una visión confortable durante un tiempo prolongado. 
Puesto que antes de la sesión los alumnos ya habían leído los textos en casa, en clase se dividen en dos grupos de 4 alumnos para que así discutan y comenten entre ellos el papel de la acomodación y musculatura extraocular en la eficiencia de la visión.

La dinámica de trabajo en grupo (30 min.) resultó de máxima discusión y concentración. La puesta en común que a continuación se realizó (30 min.) fue enriquecedora para ambos grupos. Durante el debate general había parte de alumnos que defendía que sólo la musculatura ciliar afectaba la eficiencia del sistema visual, otra parte defendía que era sólo competencia de la musculatura extraocular y el resto pensaba que había una estrecha relación entre ambos sistemas, acomodativo y vergencial. Tras 30 min. de debate aporte explicaciones partiendo de lo que ellos ya sabían.

Tras un descanso de 15 min. se plantea la lectura de un texto que trata sobre las variables que han de considerarse a la hora de evaluar acomodación y visión binocular ".Is there any evidence for the validity of diagnostic criteria used for accommodative and nonstrabismic binocular dysfunctions?" (Cacho-Martínez, García-Muñoz, \& Ruiz-Cantero, 2014). Se procede de la misma forma anteriormente descrita y se vuelve a discutir sobre la necesidad de evaluar sólo alguna o todas las variables en los pacientes según sintomatología que presenten.

De esta forma se deja atrás el modelo tradicional de enseñanza y se da paso al aprendizaje individual, puesta en común de lo aprendido y aprendizaje colaborativo.

Durante la segunda sesión se discuten casos clínicos que se plantean en clase. A cada alumno le doy un informe que describe queja del paciente, motivo de la consulta, valores de una serie de variables, antecedentes de 
enfermedades oculares y sistémicas además de la medicación que actualmente toma. Les indico una lectura que les puede ayudar, "Do we really know the prevalence of accomodative and nonstrabismic binocular dysfunctions?"(Cacho-Martínez, García-Muñoz, \& Ruiz-Cantero, 2010). Procedemos de igual forma que en la sesión anterior, pero en esta ocasión el debate es mucho más enriquecedor y activo. Las opiniones más numerosas e intensas.

Durante mi intervención les hablo de casos reales que trato en mi trabajo, los relaciono con los casos clínicos que les propongo y finalmente llegan a un diagnóstico muy próximo a la disfunción que presenta el paciente.

\section{Evaluación del aprendizaje de los estudiantes}

Para la evaluación se elaboró un cuestionario con 14 preguntas. En el cuestionario planteo tres problemas que incluyen tres aspectos distintos (saber, saber hacer y actitud). Analizo los informes y la evolución de sus respuestas. Establezco tres niveles de respuesta para cada problema. El nivel 3 se corresponde con el nivel que pretendo enseñar.

Las respuestas antes de iniciar el CIMA, en general se basaban en el conocimiento previo que tenían pues son alumnos de cuarto curso. Con el desarrollo de las dos sesiones, evolucionan las ideas en el sentido de saber interpretar los datos del examen optométrico, relacionar las variables que definen los dos sistemas acomodativo y vergencial, emitir un diagnóstico y prevención.

Al final del CIMA la mayoría de las repuestas se encontraban en el nivel 3, considerando éste como el más avanzado (figura 2,3 y 4).

Ciclos de Mejora en el Aula (2020). Experiencias de Innovación Docente de la US Esta obra se distribuye con la licencia Creative Commons 


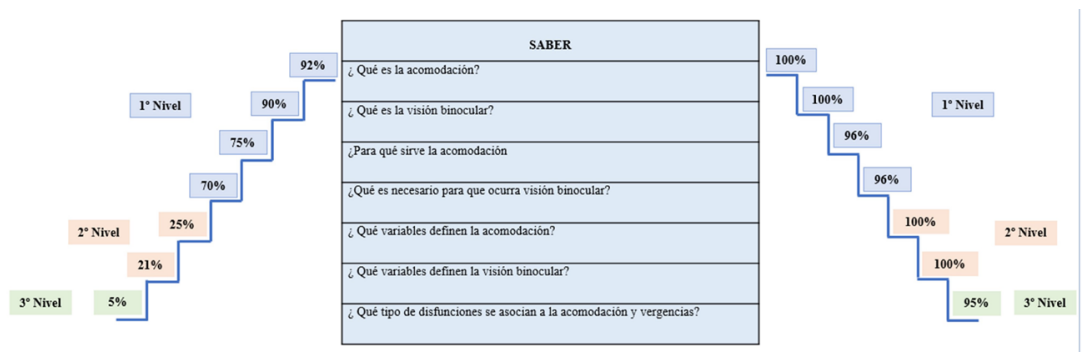

Figura 2. Escalera 1.

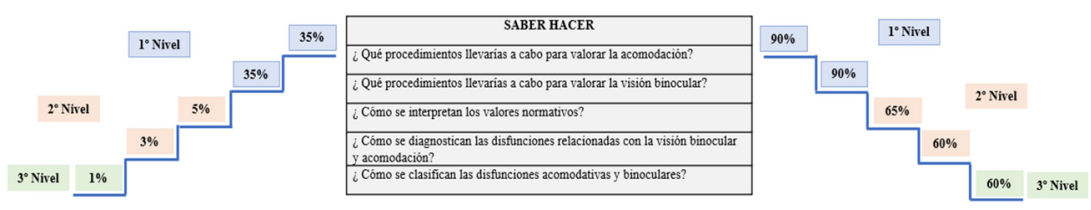

Figura 3. Escalera 2.

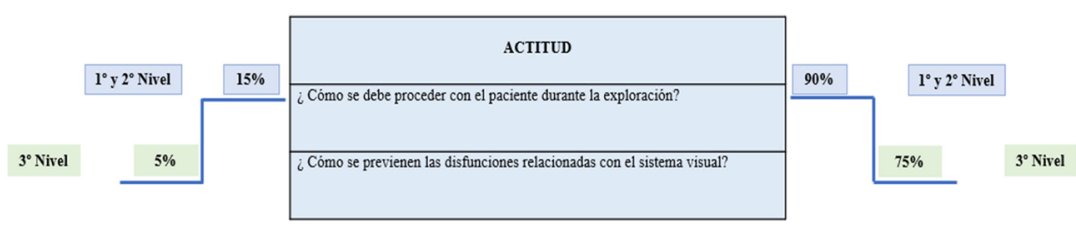

Figura 4. Escalera 3.

\section{Valoración de la experiencia}

Por mi parte he de decir que ha resultado una experiencia enriquecedora. Durante las dos sesiones he tenido una comunicación y tutorización continua con los alumnos. Cierto es que el CIMA lo he impartido en sesiones de prácticas, con alumnos de cuarto y grupos muy reducidos, situación que me ha facilitado mucho la aplicación de éste. Por parte de los alumnos, ellos me han agradecido estas dos sesiones de prácticas, me han hecho saber que es más fácil entender de esta forma conceptos que en clase se les intenta explicar.

Ciclos de Mejora en el Aula (2020). Experiencias de Innovación Docente de la US Esta obra se distribuye con la licencia Creative Commons 
Tras este curso de formación creo necesario incluir en la docencia de mis próximos cursos los siguientes aspectos:

Realizar un cuestionario inicial y final para situar el punto de partida de mi docencia y punto final del aprendizaje de los alumnos. Las respuestas deben ser agrupadas, analizadas y debo extraer conclusiones de ellas.

Debo, además, analizar las ideas de los estudiantes, mediante escaleras de aprendizaje, de esta forma aprecio la evolución en el aprendizaje y puedo ajustar los contenidos y las secuencias de actividades y organizar y elaborar un mapa de contenidos de cada uno de los bloques temáticos.

Plantear en cada bloque una serie de preguntas para conseguir un proceso de indagación sobre el tema planteado (Finkel, 2008). Preguntas que motiven la curiosidad, conseguir que el alumno entienda el significado de la pregunta, debe establecerse un compromiso intelectual con el alumno (animarlos a comparar, aplicar, analizar y sintetizar, pero nunca solamente a escuchar y recordar), favorecer el entorno para que el alumno responda las preguntas planteadas, y por último dejar a los estudiantes con una pregunta (¿Cuál es la próxima pregunta?, ¿Qué podemos preguntar ahora?,¿Qué piensas tú?........)

Incentivar el trabajo en grupo, de forma que las preguntas planteadas generen un razonamiento en el grupo, aún si el grupo se desvía de la respuesta correcta (Finkel, 2008). En el trascurso de un taller los estudiantes pueden participar en varios grupos diferentes, los grupos pequeños se pueden unir y formar grupos más grandes y los más grandes se pueden dividir y formar grupos más pequeños y se pueden alternar fases de trabajo individual y en grupo.

Ciclos de Mejora en el Aula (2020). Experiencias de Innovación Docente de la US Esta obra se distribuye con la licencia Creative Commons 
Tutorización continua del trabajo ya que, no todos los alumnos trabajan de manera uniforme. Este modelo de aprendizaje puede generar algún inconveniente ya que no todos los estudiantes trabajan de igual forma. Habrá además estudiantes que serán dominados por uno o varios miembros del grupo, otros se influenciarán por ideas equivocadas y el trabajo del grupo podría fracasar. Por ello es imprescindible la figura del profesor que supervisará en todo momento y de manera discreta el trabajo del grupo.

Debates entre los distintos grupos y con el profesor para contrastar las opiniones. Al estudiante le gustará saber a qué conclusiones han llegado otros grupos, cómo son contrastadas con las de su propio grupo, qué piensa el profesor de todas las conclusiones y finalmente querrá saber cuál es la respuesta correcta.

Ciclos de Mejora en el Aula (2020). Experiencias de Innovación Docente de la US Esta obra se distribuye con la licencia Creative Commons 
Palabras clave: Consultas de Optometría, Grado en Óptica y Optometría, Docencia Universitaria, Experimentación Docente Universitaria, Caso Clínico.

Keywords: Optometry Consultations, Optics and Optometry Degree, University Teaching, University Teaching Innovation, Clinical Case.

\section{Referencias bibliográficas}

Cacho-Marțínez, P., Cañtó-Cerdán, M., Carbonell-Bonete, S., \& García-Muñoz, Á. (2015). Characterization of Visual Symptomatology Associated with Refractive, Accommodative, and Binocular Anomalies. Journal of Ophthalmology, 895803.

Cacho-Martínez, P., García-Muñoz, Á., \& Ruiz-Cantero, M. T. (2010). Do we really know the prevalence of accomodative and nonstrabismic binocular dysfunctions? Journal of Optometry, 3(4), 185-197.

Cacho-Martínez, P., García-Muñoz, Á., \& Ruiz-Cantero, M. T. (2014). Is there any evidence for the validity of diagnostic criteria used for accommodative and nonstrabismic binocular dysfunctions? Journal of Optometry, 7(1), 2-21.

Finkel, D. (2008). Dar clase con la boca cerrada. Universitat de Valencia: Servei de publicacions.

García-Muñoz, Á., Carbonell-Bonete, S., Cantó-Cerdán, M., \& Cacho-Martínez, P. (2016). Accommodative and binocular dysfunctions: prevalence in a randomised sample of university students. Clinical \& Experimental Optometry, 99(4), 313-321.

Bain, K. (2007). Lo que hacen los mejores profesores universitarios. Universitat de Valencia. Servei de publicacions.

De Alba, N. y Porlán, R. (2017). La metodología de enseñanza. En R. Porlán (Coord.), Enseñanza Universitaria. Cómo mejorarla (pp. 37-51). Madrid: Morata.

Ciclos de Mejora en el Aula (2020). Experiencias de Innovación Docente de la US Esta obra se distribuye con la licencia Creative Commons 